\section{Simultaneous Velocity and Position Estimation via Distance-only Measurements with Application to Multi-Agent System Control}

\author{
Bomin Jiang, Mohammad Deghat, Member, IEEE and \\ Brian D.O. Anderson, Life Fellow, IEEE
}

\begin{abstract}
This paper proposes a strategy to estimate the velocity and position of neighbor agents using distance measurements only. Since with agents executing arbitrary motions, instantaneous distance-only measurements cannot provide enough information for our objectives, we postulate that agents engage in a combination of circular motion and linear motion. The proposed estimator can be used to develop control algorithms where only distance measurements are available to each agent. As an example, we show how this estimation method can be used to control the formation shape and velocity of the agents in a multi agent system.
\end{abstract}

\section{INTRODUCTION}

The performance of multi-agent systems in various tasks, e.g. consensus [1], formation shape control [2], cooperative geolocalization [3], etc. has been studied with increasing intensity over recent years. These tasks are usually required to be performed in a decentralized way [4] and using limited information. Examples of these tasks are retrieving information from an area covered by a sensor network (where the agents are sensors deployed in the area) [5], or moving together in a desired formation shape from one point to another.

In a formation control problem, which is the focus of this paper, each agent tries to contribute to achieve the global goal of the formation using measurements of, typically, relative position and velocity of its neighbors. Examples of such problems are given in [6], [7]. These problems become more challenging when the agents cannot instantaneously measure all the information required to apply motion corrections to achieve the final goal of the formation and have to estimate some of this information using their measurements.

An example of such a challenging problem is given in [8], where a formation (shape and translation motion) control method, called stopand-go, has been devised to control the agents not able to measure the relative positions (both distance and angle) of their neighbors, but only able to measure the distances to their respective neighbors. This measurement restriction makes the control problem significantly harder.

This paper treats a related problem. Agents are required to estimate the relative position and velocity of their neighbor agents using only distance measurements to the neighbors, and achieve both velocity consensus and formation shape control. The key is to postulate that the motion of each agent comprises two parts: a translation and a circular motion. The circular motion is around a moving center (so it can appear sinusoidal), and it is the centers of each agent's motion, rather than the agents themselves, which achieve velocity consensus. The purpose of the superimposed circular motion is to allow interagent localization and velocity estimation, not using instantaneous measurements, but using distance measurements collected over an

\footnotetext{
*This work is supported by National ICT Australia, which is funded by the Australian Research Council through the ICT Centre of Excellence program.

Bomin Jiang is with Department of Mechanical Engineering, Massachusetts Institute of Technology. Mohammad Deghat is with the School of Engineering and Information Technology, the University of New South Wales. Brian D. O. Anderson is with Research School of Engineering, The Australian National University and National ICT Australia. Bomin Jiang and Mohammad Deghat were previously with the ANU and NICTA. (e-mail: bominjemit.edu, m.deghateunsw.edu.au, Brian.Andersoneanu.edu.au)
}

interval. We postulate that neighbor agents remain in communication even if they initially have different velocities.

The notion of using deliberate motions of agents to assist in localization was suggested in [9], in relation to sensor network localization. However, the motions in [9] are random. This paper studies the localization problem using distance-only measurements when agents are executing independent circular motions. Note that the idea of introducing sinusoidal perturbation in formation control problems is not wholly novel: in [10], the authors have introduced sinusoidal perturbations to the usual gradient based control algorithm in order to achieve a different objective. An advantage of having a combination of linear and circular motion over only linear motion as in [8] is that the agents are less likely to travel out of communication range during the localization process.

An abbreviated conference version of this paper has been presented in [11]. The novel contributions of the paper, in comparison to the conference paper [11], are as follows (a) proposing a discrete-time control algorithm to achieve velocity consensus and simultaneous formation shape control, usable with distance-only measurements, and (b) introducing a major improvement by adaptively adjusting the circular motion radius.

The rest of this paper is organized as follows. Section II gives a solution to the location and velocity estimation problem using distance-only measurements when each agent is executing a combination of linear motion and circular motions. Section III discusses an improvement of the algorithm derived in the previous section involving adaptively adjusting the circular motion radius. Section IV discusses a discrete time control algorithm to achieve velocity consensus and formation shape control with distance-only measurements. Simulations are included in each section. Concluding remarks and directions for future research are given in Section V.

\section{RELATIVE POSITION AND VELOCITY ESTIMATION USING SINUSOIDAL PERTURBATION}

In the conference version of this paper [11], we gave detailed explanation on how to infer a neighboring agent's relative position and velocity. We have also discussed all special cases. Here we just give a brief introduction to the ideas.

\section{A. Problem statement}

Consider two point agents, 1 and 2. Each agent performs a combination of circular and rectilinear motion, so each has a certain radius, direction and angular velocity for the circular motion and velocity for the rectilinear motion. Agent 1 knows its own radius, angular velocity and the translational velocity of its circle center and can only measure (continuously) the distance but not bearing of agent 2 . Conversely, agent 2 knows its radius, angular velocity and the velocity of its circle center and can only measure the distance of agent 1 . The goal is for both agents to localize and sense the velocities of each other for velocity consensus purposes.

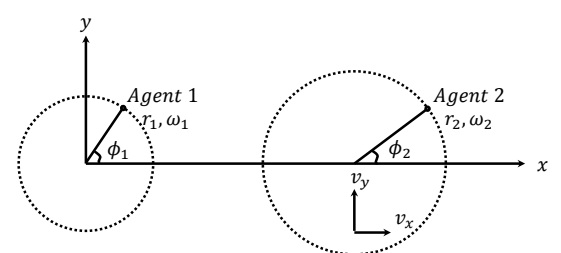

Fig. 1. Set up a coordinate system with respect to agent 1's circle center

As shown in Fig. 1, we set up a global coordinate system with origin at agent 1 's circle center and agent 2 's circle center on the $x$ axis when $t=0$. Suppose $r_{i}$ is the radius of agent $i$ 's motion,

Preprint submitted to IEEE Transactions on Automatic Control. Received: September 21, 2015 14:00:50 PST 
$\omega_{i}$ is the angular velocity of agent $i, z(t)$ is the distance at time $t$ between agent 1 and 2 and $d$ is the distance between the two circle centers. The coordinate system is defined by the agent pair, and is used for analysis purposes by us. Its orientation with respect to agent 1 's local coordinate basis is not known by agent 1 at this stage though the orientation can be obtained after that agent learns $\phi_{1}$. In addition, let $v_{c_{i}}$ be the velocity of agent $i$ 's circle center, $v_{j i}$ be the relative velocity of agent $j$ 's circle center with respect to agent $i$ 's circle center, $v_{x}$ be the $x$ component of the velocity $v_{21}$, and $v_{y}$ be the $y$ component of the velocity $v_{21}$. The positive direction of angular velocities is counter-clockwise.

We assume in this paper that $v_{x}$ and $v_{y}$ are constant for $k T<$ $t<(k+1) T, T>0, k=0,1,2, \cdots$ and may only change at time instants $k T$, perhaps reflecting a discrete-time consensus algorithm. We explain later how to choose $T$.

There holds

$$
\begin{aligned}
z^{2}(t)= & {\left[d+v_{x} t+r_{2} \cos \left(\omega_{2} t+\phi_{2}\right)-r_{1} \cos \left(\omega_{1} t+\phi_{1}\right)\right]^{2} } \\
& +\left[v_{y} t+r_{2} \sin \left(\omega_{2} t+\phi_{2}\right)-r_{1} \sin \left(\omega_{1} t+\phi_{1}\right)\right]^{2}
\end{aligned}
$$

Let $d_{x}=d+v_{x} t$ and $d_{y}=v_{y} t$ and rewrite (1) using easy algebra as:

$$
\begin{aligned}
z^{2}(t)= & \left(d_{x}^{2}+d_{y}^{2}+r_{1}^{2}+r_{2}^{2}\right) \\
& +2 d_{x} r_{2} \cos \left(\omega_{2} t+\phi_{2}\right)+2 d_{y} r_{2} \sin \left(\omega_{2} t+\phi_{2}\right) \\
& -2 d_{x} r_{1} \cos \left(\omega_{1} t+\phi_{1}\right)-2 d_{y} r_{1} \sin \left(\omega_{1} t+\phi_{1}\right) \\
& -2 r_{1} r_{2} \cos \left[\left(\omega_{1}-\omega_{2}\right) t+\left(\phi_{1}-\phi_{2}\right)\right]
\end{aligned}
$$

\section{B. Finding relative position and velocity of a neighbor}

In the system comprising a pair of agents 1 and 2, without loss of generality, we only show how agent 1 can localize and estimate the relative velocity of agent 2 . The first step is for agent 1 to identify the angular velocity of agent 2, using a Fourier representation of $z^{2}(t)$ for $t \in[0, T]$. When $\left\|v_{21}\right\| T$ is sufficiently small in comparison to $r_{1}, r_{2}$ and $d$, four distinct peaks will show up at $0,\left|\omega_{1}\right|,\left|\omega_{2}\right|$ and $\left|\omega_{1}-\omega_{2}\right|$ in frequency domain. This allows agent 1 to pick up the angular velocity of agent 2 . More insights about this assumption will be discussed in Section III.

In order to identify the value of $d, \phi_{1}, v_{x}$ and $v_{y}$, we allow agent 1 to measure the distance between the two agents $z(t)$ and then analyze the Fourier series of the periodic extension of $z^{2}(t)$. Theorem 1 gives the procedure to identify $d, \phi_{1}, v_{x}$ and $v_{y}$; detailed explanation about this process is given in [11].

Theorem 1. For a pair of point agents in $\mathbb{R}^{2}$, if each agent is executing a combination of circular motion and linear motion (as described by (1) and (2)) and the associated angular frequencies are commensurate, each agent can find the position and translational velocity of the other agent by distance-only measurements over an interval, which equals to an integer multiple of a common period for the agent's circular motions ${ }^{1}$.

Proof. The definitions of $r_{1}, r_{2}, \omega_{1}, \omega_{2}, d, z, v_{x}, v_{y}, \phi_{1}$ and $\phi_{2}$ are the same as in Section II-A. We choose $T$ so that there exist integers $k_{1}, k_{2}$ defining the multiple which $T$ represents of the periods associated with the two angular velocities, i.e. $k_{1}=\frac{\omega_{1} T}{2 \pi}$ and $k_{2}=\frac{\omega_{2} T}{2 \pi}$. The existence of $k_{1}$ and $k_{2}$ relates to the concept of commensurable numbers, see Remark 3 in [11].

Suppose one continuously measures $z$ for a time period $T$ and finds the Fourier series of the periodic extension of $z^{2}$. Consider (2) and suppose $c_{n}$ are the coefficients of Fourier series using powers of $e^{j \frac{2 \pi}{T}}$ of the periodic extension of $z^{2}$. Similarly, $s_{n}, u_{n}$ and $w_{n}$ are the coefficients of the Fourier series of the periodic extension of

\footnotetext{
${ }^{1}$ Refer to Remark 3 in [11] for treatment of incommensurate periods.
}

$\left(d_{x}^{2}+d_{y}^{2}+r_{1}^{2}+r_{2}^{2}\right),-2 d_{x} r_{1} \cos \left(\omega_{1} t+\phi_{1}\right)-2 d_{y} r_{1} \sin \left(\omega_{1} t+\phi_{1}\right)$ and $2 d_{x} r_{2} \cos \left(\omega_{2} t+\phi_{2}\right)+2 d_{y} r_{2} \sin \left(\omega_{2} t+\phi_{2}\right)$ respectively. From (2) we know that for any $n>0 \cap n \neq\left|k_{1}-k_{2}\right|$ there holds $c_{n}=$ $s_{n}+u_{n}+w_{n}$. Further by calculating the Fourier Series of (2) we know for any $n>0 \cap n \neq k_{1}, k_{2}$ or $\left|k_{1}-k_{2}\right|$ there holds

$$
\begin{aligned}
c_{n} & =\frac{1}{n^{2}} R+\frac{1}{n} I j \\
& +\left(\frac{1}{n-k_{1}}+\frac{1}{n+k_{1}}\right) \cdot 2 U+\left(\frac{1}{n-k_{2}}+\frac{1}{n+k_{2}}\right) \cdot 2 W
\end{aligned}
$$

where

$$
\begin{gathered}
U=r_{1}\left(j \frac{v_{x} T}{4 \pi}+\frac{v_{y} T}{4 \pi}\right) e^{j\left(\phi_{1}+\pi\right)} \\
W=r_{2}\left(j \frac{v_{x} T}{4 \pi}+\frac{v_{y} T}{4 \pi}\right) e^{j\left(\phi_{2}\right)} \\
R=\frac{\left(v_{x}^{2}+v_{y}^{2}\right) T^{2}}{2 \pi^{2}}
\end{gathered}
$$

and

$$
I=\frac{\left(v_{x}^{2}+v_{y}^{2}\right) T^{2}+2 v_{x} d T}{2 \pi}
$$

From (3) and the non-singular condition (Lemma 3 of [11]) we know that if we have four values of $c_{n}, n>0 \cap n \neq k_{1}, k_{2}$ or $\mid k_{1}-$ $k_{2}$, we are able to find the unique solutions of $R, I, U$ and $W$.

Now we have the value of $U$ and $W$ and can obtain $u_{k_{1}}$ from the Fourier Series of (2). Furthermore, from (4) and the Fourier Series of (2) we know that

$$
\begin{aligned}
u_{k_{1}}-\frac{U}{2 k_{1}} & =\frac{r_{1}}{2}\left(d+\frac{1}{2} v_{x} T-\frac{1}{2} v_{y} T j\right) e^{j\left(\phi_{1}+\pi\right)} \\
U & =r_{1}\left(j \frac{v_{x} T}{4 \pi}+\frac{v_{y} T}{4 \pi}\right) e^{j\left(\phi_{1}+\pi\right)}
\end{aligned}
$$

and $d, v_{x}, v_{y}$ and $\phi_{1}$ can be found from these equations. They are $d=\frac{2}{r_{1}}\left|u_{k_{1}}-\frac{U}{2 k_{1}}+\pi j U\right|, \phi_{1}=\arg \left(u_{k_{1}}-\frac{U}{2 k_{1}}+\pi j U\right)+\pi$, $v_{x}=\operatorname{Im}\left(\frac{4 \pi U}{T r_{1} e^{j\left(\phi_{1}+\pi\right)}}\right)$, and $v_{y}=\operatorname{Re}\left(\frac{4 \pi U}{T r_{1} e^{j\left(\phi_{1}+\pi\right)}}\right)$

\section{AdAPtive RADiUs Assignment}

In the above sections, we let each agent infer the position and relative velocity information of neighboring agents by 1) carrying out a Fourier transform and then 2) identifying peaks to estimate $\omega$ of neighboring agents 3 ) solving the set of linear equations (3). In step 2) if $\left\|v_{12}\right\| T$ is sufficiently small in comparison to $r_{1}, r_{2}$, we can show that there are always peaks at $k_{1}$ and $k_{2}$.

Lemma 1. Adopt the hypothesis in Theorem 1. If $d>r_{1}, r_{2}$ and $\left\|v_{21}\right\| T$ is sufficiently small in comparison to $r_{1}, r_{2}$, then $c_{n}$ (regarded as a function of the integer $n$ ) has peaks at $n=k_{1}$ and $n=k_{2}$.

Proof. When $n \neq 0, k_{1}, k_{2},\left|k_{1}-k_{2}\right|$, the dependence of $\left\|c_{n}\right\|$ on $\left\|v_{12}\right\|$ can be expressed as follows

$$
\begin{aligned}
\left\|c_{n}\right\| & =\left\|h_{1}\left(k_{1}, k_{2}, T\right)\right\| v_{21}\left\|^{2}+h_{2}\left(k_{1}, k_{2}, T\right)\right\| v_{21} \| d \\
& +h_{3}\left(k_{1}, k_{2}, T\right)\left\|v_{21}\right\| r_{1}+h_{4}\left(k_{1}, k_{2}, T\right)\left\|v_{21}\right\| r_{2} \| .
\end{aligned}
$$

On the other hand, when $n=k_{1}$, there holds

$$
\begin{aligned}
\left\|c_{n}\right\| & =\left\|h_{5}\left(k_{1}, k_{2}, T\right)\right\| v_{21}\left\|^{2}+h_{6}\left(k_{1}, k_{2}, T\right)\right\| v_{21} \| d \\
& +h_{7}\left(k_{1}, k_{2}, T\right)\left\|v_{21}\right\| r_{2}+h_{8}\left(k_{1}, k_{2}, T\right) d r_{1} \|
\end{aligned}
$$

where $h_{1,2, \cdots, 8}\left(k_{1}, k_{2}, T\right)$ are all bounded functions for integer $k_{1}, k_{2}$ and $T>0$.

Now compare (10) and (11). Suppose $d>r_{1}, r_{2}$. For a sufficiently small $\left\|v_{21}\right\| T$ the term $h_{8}\left(k_{1}, k_{2}, T\right) d r_{1}$ will be dominant and thus 
there will be peak recognized at $n=k_{1}$. Similarly there will also be peak recognized at $n=k_{2}$.

According to Lemma 1 , the proposed algorithm works if $d>$ $r_{1}, r_{2}$ and $\left\|v_{21}\right\| T$ is sufficiently small. In reality, $d>r_{1}, r_{2}$ is automatically satisfied if we aim to avoid collision. Furthermore, in order to ensure that $\left\|v_{21}\right\| T$ is sufficiently small for each agent pair, we propose an adaptive radius algorithm whereby $r_{i}$ of each agent is re-set at the end of each $T$ second intervals as follows

$$
r_{i}((k+1) T)=\alpha \cdot \max _{j}\left\{\left\|v_{i j}(k T)\right\|\right\}
$$

where $j$ denotes the indices of neighboring agents of $i$ and $\alpha$ is a sufficient large value. Note that because $r_{i}$ only changes at the end of each interval $T$, the radius is fixed within each interval.

The adaptive radius law will ensure that $r_{1}, r_{2}>\alpha \cdot\left\|v_{12}\right\|$ holds for each agent pair. Furthermore, as velocity consensus is being achieved, $\left\|v_{i j}(k T)\right\|$ will approach zero and so will $r_{i}$. It is noticeable that the accuracy of estimation of $\left\|v_{i j}(k T)\right\|$ is independent of the value of radius. Each agent can estimate the norm of velocities of neighbors' circle centers via $R$ according to (6), even if no peaks are identified. This phenomenon is consistent with the paper [8], which shows that without circular motions, for agents only doing linear motions, it is possible to estimate the norm of relative velocities of neighbors, even though the directions are left unknown.

When there are sudden changes in velocities of agents due to e.g. wind or deliberate change of course by a leader agent, an already achieved consensus and formation may be broken. In this case, even if the radius of the circle of each agent has already approached to zero, each agent can still obtain a good estimate of the absolute value of velocities of its neighbors' circle centers. This can result in an increase of radius of circular motions in response to the broken consensus, which allows the agents to achieve velocity consensus and formation shape control again.

Similarly to the setting in Section V-B in [11], consider a multiagent system shown in Fig. 2, suppose $\omega_{i}$ is the angular velocity of agent $i, T$ is the sampling time interval, $\left(v_{x i}, v_{y i}\right)$ is the translational velocity of agent $i$ and $\left(p_{x i}, p_{y i}\right)$ is the position of circle center of agent $i$. In the simulation, we set $\omega_{1}=\omega_{3}=5, \omega_{2}=-3$, $T=2 \pi$. When $t=0,\left(v_{x 1}, v_{y 1}\right)=(-4,2),\left(v_{x 2}, v_{y 2}\right)=(3,-2)$, $\left(v_{x 3}, v_{y 3}\right)=(2,4),\left(p_{x 1}, p_{y 1}\right)=(70,30),\left(p_{x 2}, p_{y 2}\right)=(0,50)$ ,$\left(p_{x 3}, p_{y 3}\right)=(0,0)$ and $\varepsilon=0.35$. Fig. 3 and Fig. 4 shows the simulation result, where the velocity of agent 2 changes suddenly at $t=20 T$.

Fig. 2. Topology of the multi-agent system

\section{COMbining Velocity CONSEnsus And Formation SHAPE CONTROL}

\section{A. Stability of discrete time control algorithm}

To be best of our knowledge, there is so far no discrete-time algorithm that combines velocity consensus with formation shape control for a multi-agent system. Although there is a continuous-time version algorithm proposed in [12], it cannot be implemented directly when we let each agent measure distance for a time period $T$ and then make a velocity adjustment at the end of each such interval. This section proposes a discrete-time algorithm that combines velocity consensus with formation shape control, where the sampling time interval is not required to be sufficiently small.

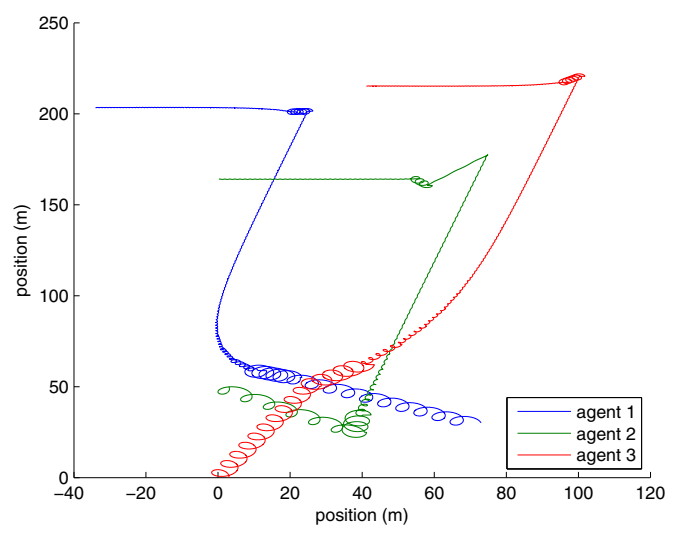

Fig. 3. Trajectories of three agents. The radius are large at the beginning but reduced as velocity consensus is achieved. At $\mathrm{t}=20 \mathrm{~T}$, the radius of circular motions of agents increases temporarily in response to the broken consensus.
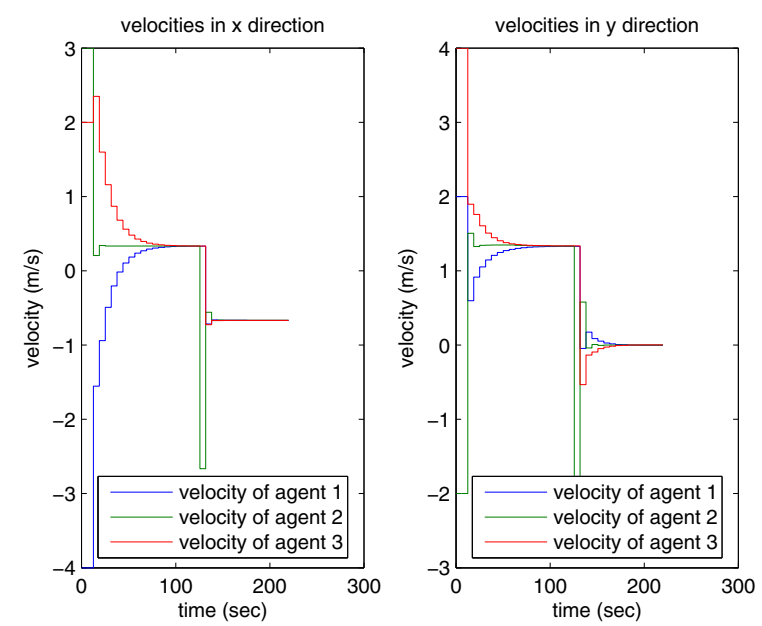

Fig. 4. Re-achieved consensus: there is a sudden change in velocity of agent 2 at $t=20 T$. After that, the translational velocities achieves consents again.

We start with the continuous time algorithm as stated in [12]:

$$
\begin{aligned}
& \dot{p}_{i}=v_{i} \\
& \dot{v}_{i}=\sum_{j \in \mathcal{N}_{i}}\left(v_{j}-v_{i}\right)+2 \sum_{j \in \mathcal{N}_{i}}\left(d_{i j}^{* 2}-d_{i j}^{2}\right)\left(p_{i}-p_{j}\right)
\end{aligned}
$$

where $p_{i}$ is the position of the $i$ th agent, $i=1, \cdots, N, v_{i}$ is the velocity of the $i$ th agent and $\mathcal{N}_{i}$ is the set of neighboring agents of agent $i$. Further, $d_{i j}^{*}$ is the desired distance between agent $i$ and $j$ and $d_{i j}$ is the current distance between agent $i$ and $j$. In our context, agent positions and velocities refer to the center of the circular motion. The system equations (13) can be written in the matrix form

$$
\begin{aligned}
& \dot{p}=v \\
& \dot{v}=-\left(\mathcal{L} \otimes I_{2}\right) v+f(p)
\end{aligned}
$$

where $p \in \mathbb{R}^{2 N}$ is the vector of all $p_{i}$ stacked together, $\mathcal{L}$ denotes the Laplacian matrix which is positive semi-definite and has one zero eigenvalue when the graph is connected and undirected, and $f(p)$ is a vector with the entries $2 \sum_{j \in \mathcal{N}_{i}}\left(d_{i j}^{* 2}-d_{i j}^{2}\right)\left(p_{i}-p_{j}\right), i=1, \cdots, N$. A discrete version of (13) for our use is given by

$$
\begin{aligned}
& \dot{p}_{i}=v_{i} \\
& v_{i}((k+1) T)=v_{i}(k T)+\epsilon_{1} T \sum_{j \in \mathcal{N}_{i}}\left(v_{j}(k T)-v_{i}(k T)\right) \\
& \quad+2 \epsilon_{2} T \sum_{j \in \mathcal{N}_{i}}\left(d_{i j}^{* 2}-d_{i j}^{2}(k T)\right)\left(p_{i}(k T)-p_{j}(k T)\right)
\end{aligned}
$$

Preprint submitted to IEEE Transactions on Automatic Control. Received: September 21, 2015 14:00:50 PST 
where $\epsilon_{1}, \epsilon_{2}$ are suitably small positive constants; more information is given below. Note that the first equation remains in continuous time while the second equation is discretized. However, since $v_{i}(t)$ is constant over an interval $T$, it follows that the discretization of the first equation, viz. $p_{i}((k+1) T)=p_{i}(k T)+T v_{i}(k T)$ exactly interpolates the continuous function $p_{i}(t)$ for $t=k T$ with integer $k$.

To show the stability of (15), we start with the continuous-time system and make the following transformation

$$
\bar{p}_{r}=R p, \quad \bar{v}_{r}=R v
$$

where $R$ is an orthonormal matrix whose first two rows are $(\mathbf{1} \otimes$ $\left.I_{2}\right)^{\top} / \sqrt{N}, \bar{p}_{r}:=\left[\begin{array}{ll}p_{0}^{\top} & \bar{p}^{\top}\end{array}\right]^{\top}$ with $p_{0} \in \mathbb{R}^{2}$ and $v:=\left[\begin{array}{ll}v_{0}^{\top} & \bar{v}^{\top}\end{array}\right]^{\top}$ with $v_{0} \in \mathbb{R}^{2}$. Then $\dot{v}_{0}=0$, that is the position of the center of mass of the agents in $\bar{p}$-coordinates is constant, and the system equations in $\bar{p}$ and $\bar{v}$ are

$$
\begin{aligned}
& \dot{\bar{p}}=\bar{v} \\
& \dot{\bar{v}}=L \bar{v}+\bar{f}(\bar{p})
\end{aligned}
$$

where $L$ is the $(2 N-2) \times(2 N-2)$ nonzero block of $-R\left(\mathcal{L} \otimes I_{2}\right) R^{\top}$ which is negative definite and $\bar{f}(\bar{p})$ contains the nonzero entries of $R f\left(R^{\top} \bar{p}\right)=R f(p)$.

Our approach to show the stability of (15) is as follows: first we define a Malkin structure in Definition 1. After that we show in Lemma 2 that (17) is transformable to a Malkin structure. Then we develop in Theorem 2 a discrete-time version of the continuous-time Malkin's theorem as invoked by Krick [13]. Finally, we use these results and show in Theorem 3 that (15) is stable for sufficiently small values of $\epsilon_{1}$ and $\epsilon_{2}$.

Definition 1. (Malkin structure) ${ }^{2}$

A system has Malkin structure if it is in the form

$$
\dot{r}=\left[\begin{array}{ll}
0 & 0 \\
0 & A
\end{array}\right] r+g(\theta, \rho), r=\left[\begin{array}{l}
\theta \\
\rho
\end{array}\right], g=\left[\begin{array}{c}
\Theta(\theta, \rho) \\
P(\theta, \rho)
\end{array}\right]
$$

where $A$ is constant with negative real part eigenvalues. Furthermore, $g(\theta, \rho)$ is a second order term satisfying the following conditions i) $g(\theta, 0)=0$, ii) there exists

$$
\begin{gathered}
h_{1}(\theta)=\lim _{\rho \rightarrow 0} \frac{\Theta(\theta, \rho)}{\|\rho\|}, \quad h_{2}(\theta)=\lim _{\rho \rightarrow 0} \frac{P(\theta, \rho)}{\|\rho\|}, \\
b_{1}=\left\{\begin{array}{c}
\frac{\Theta(\theta, \rho)}{\|\rho\|} \text { if } \rho \neq 0 \\
h_{1}(\theta) \text { if } \rho=0
\end{array}, b_{2}=\left\{\begin{array}{c}
\frac{P(\theta, \rho)}{\|\rho\|} \text { if } \rho \neq 0 \\
h_{2}(\theta) \text { if } \rho=0
\end{array}\right.\right.
\end{gathered}
$$

such that $b_{1}$ and $b_{2}$ are bounded smooth functions and $b_{2}(0)=0$;

Lemma 2. The system equations in (17) can be transferred to a Malkin structure through a local diffeomorphism around the equilibrium point of (17).

Proof. Suppose there are $N$ agents in a formation. Consider the single-integrator formation shape control system [14]

$$
\dot{p}=f(p)
$$

where $f(p)$ is a vector with entries

$$
\sum_{j \in \mathcal{N}_{i}}\left(d_{i j}^{* 2}-d_{i j}^{2}\right)\left(p_{i}-p_{j}\right), \quad i=1 . \cdots, N
$$

and $d_{i j}, d_{i j}^{*}, p_{i}$ and $p_{j}$ are as defined in (13). It is shown in [13] that there is a local diffeomorphism around the equilibrium point that transfers (19) to a Malkin structure. Suppose the diffeomorphism is

$$
r=\phi(\bar{p}), \quad \bar{p}=\psi(r)
$$

\footnotetext{
${ }^{2}$ There are minor differences in the definition of Malkin structure in
} different references. We use the definition in [13] here. such that $\dot{\bar{p}}=\bar{f}(\bar{p})$, where $\bar{p}$ and $\bar{f}$ are defined in (16) and (17), transfers to a Malkin structure

$$
\dot{r}=\left[\begin{array}{ll}
0 & 0 \\
0 & A
\end{array}\right] r+g(\theta, \rho), r=\left[\begin{array}{l}
\theta \\
\rho
\end{array}\right], g=\left[\begin{array}{c}
\Theta(\theta, \rho) \\
P(\theta, \rho)
\end{array}\right]
$$

where $A$ has eigenvalues with negative real parts and $g(\theta, \rho)$ fulfills the conditions of the second order term $g(\cdot)$ in Definition 1. Let $n_{\theta}$ denote the number of elements in $\theta$ and $n_{\rho}$ denote the number of elements in $\rho$.

Now we are going to show that the velocity and formation shape control problem in (17)

$$
\ddot{\bar{p}}=L \dot{\bar{p}}+\bar{f}(\bar{p}), L=L^{T}<0
$$

is also transferred to a Malkin structure by the same diffeomorphism. Observe

$$
\dot{r}=\frac{\partial \phi}{\partial p} \dot{\bar{p}}=\frac{\partial \phi}{\partial p} \bar{f}(\bar{p})=\left(\frac{\partial \psi}{\partial r}\right)^{-1} \bar{f}(\psi(r)) .
$$

Now the right sides of (21) and (23) are the same. Next

$$
\ddot{r}=\Phi(\bar{p}, \dot{\bar{p}})+\frac{\partial \phi}{\partial p} \ddot{\bar{p}}
$$

where the row $i$ column $j$ entry of $\Phi(\bar{p}, \dot{\bar{p}})$ takes the form

$$
\sum_{k} \frac{\partial^{2} \phi_{i}}{\partial \bar{p}_{j} \partial \bar{p}_{k}} \dot{\bar{p}}_{j} \dot{\bar{p}}_{k}
$$

Combining (24) and (22), we obtain

$$
\ddot{r}=\Psi(r, \dot{r})+\frac{\partial \phi}{\partial \bar{p}} L \dot{\bar{p}}+\frac{\partial \phi}{\partial \bar{p}} \bar{f}(\bar{p})
$$

where $\Psi(r, \dot{r})=\Phi\left(\psi(r), \frac{\partial \phi}{\partial r} \dot{r}\right)$ is $O\left(\|\dot{r}\|^{2}\right)$. Hence

$$
\ddot{r}=\Psi(r, \dot{r})+\left(\frac{\partial \psi}{\partial r}\right)^{-1} L\left(\frac{\partial \psi}{\partial r}\right) \dot{r}+\left[\begin{array}{cc}
0 & 0 \\
0 & A
\end{array}\right] r+g(\theta, \rho) .
$$

Now we have the system equation

$$
\frac{d}{d t}\left[\begin{array}{l}
r \\
\dot{r}
\end{array}\right]=C\left[\begin{array}{l}
r \\
\dot{r}
\end{array}\right]+\left[\begin{array}{c}
0 \\
\Psi(r, \dot{r})+h \cdot \dot{r}+g(\theta, \rho)
\end{array}\right]
$$

where

$$
\begin{gathered}
C=\left[\begin{array}{cc}
0 & \left.\begin{array}{cc}
0 & 0 \\
0 & A
\end{array}\right] \\
\left(\frac{\partial \psi}{\partial r}\right)^{*-1} L\left(\frac{\partial \psi}{\partial r}\right)^{*}
\end{array}\right] \\
h=-\left(\frac{\partial \psi}{\partial r}\right)^{*-1} L\left(\frac{\partial \psi}{\partial r}\right)^{*}+\left(\frac{\partial \psi}{\partial r}\right)^{-1} L\left(\frac{\partial \psi}{\partial r}\right)
\end{gathered}
$$

where $^{*}$ denotes the value at system equilibrium. Observe that $C$ is constant and takes the following form

$$
C=\left[\begin{array}{ll}
0 & D \\
0 & E
\end{array}\right]
$$

with $E$ a $\left(n_{\theta}+2 n_{\rho}\right) \times\left(n_{\theta}+2 n_{\rho}\right)$ nonsingular square matrix.

Consider the nonsingular similarity transformation $T=$ $\left[\begin{array}{cc}I & -D E^{-1} \\ 0 & I\end{array}\right]$ and define $\left[\begin{array}{c}\bar{r} \\ \dot{r}\end{array}\right]=T\left[\begin{array}{l}r \\ \dot{r}\end{array}\right]$. Note $\bar{r}=\left[\begin{array}{c}\bar{\theta} \\ \bar{\rho}\end{array}\right]$. There holds

$$
\left.\frac{d}{d t}\left[\left[\begin{array}{c}
\bar{\theta} \\
\bar{\rho} \\
\dot{r}
\end{array}\right]\right]=\left[\begin{array}{ll}
0 & 0 \\
0 & E
\end{array}\right]\left[\begin{array}{c}
\bar{\theta} \\
\bar{\rho} \\
\dot{r}
\end{array}\right]\right]+T \cdot o(\bar{r}, \dot{r})
$$

with $\left[\begin{array}{ll}0 & 0 \\ 0 & E\end{array}\right]=T C T^{-1}$.

Krick shows in [13] that first applying the diffeomorphism $r=$ $\phi(\bar{p})$ and then linearizing the system is equivalent with first linearizing the system and then applying the diffeomorphism. It is shown in [15] that the system (22) is locally exponentially stable on 
a center manifold; therefore the system matrix of the linearization of (22) at a point on the center manifold $\left[\begin{array}{cc}0 & I \\ \left(\frac{\partial \bar{f}}{\partial \bar{p}}\right)^{*} & L\end{array}\right]$ has eigenvalues with non-positive real parts. Furthermore, because the local diffeomorphism is smooth, its linearization around the equilibrium $\left[\begin{array}{cc}\frac{\partial \phi}{\partial p} & 0 \\ 0 & \frac{\partial \phi}{\partial p}\end{array}\right]$ is a non-singular similarity transformation. Therefore, the linearization of the system equation after applying the diffeomorphism

$$
C=\left[\begin{array}{cc}
\frac{\partial \phi}{\partial p} & 0 \\
0 & \frac{\partial \phi}{\partial p}
\end{array}\right]\left(\left[\begin{array}{cc}
0 & I \\
\left(\frac{\partial \bar{f}}{\partial \bar{p}}\right)^{*} & L
\end{array}\right]\right)\left[\begin{array}{cc}
\frac{\partial \phi}{\partial p} & 0 \\
0 & \frac{\partial \phi}{\partial p}
\end{array}\right]^{-1}
$$

also has eigenvalues with non-positive real parts. With (28) and the fact that $A$ and $L$ are both full rank, we know $E$ is a non-singular square matrix. Thus $E$ has eigenvalues with negative real parts.

Define

$$
l(\bar{\theta}, \bar{\rho}, \dot{r})=o(\bar{r}, \dot{r})=\Psi(r, \dot{r})+h \cdot \dot{r}+g(\theta, \rho)
$$

Because

$\Psi$ is $O\left(\|\dot{r}\|^{2}\right)$

$h=0$ when $\bar{\rho}=0$ and $\dot{r}=0$

$g(\theta, \rho)$ fulfills the conditions of second order term $g(\cdot)$ in Definition 1

we can conclude that $l(\bar{\theta}, \bar{\rho}, \dot{r})$ fulfills the conditions of second order term $g(\cdot)$ in Definition 1 . Therefore, we have completed the proof.

Theorem 2. Consider the time-discretized version of (30) below of the Malkin structure in Definition 1 , where $\theta_{k}$ and $\rho_{k}$ are the kth sample of the quantities $\theta$ and $\rho$ in Definition 1. Then there exists a sufficiently small sampling time interval $\epsilon$ (certainly with $\epsilon<1$ ), and a sufficiently small open ball $\mathcal{V}$ around the origin such that if $\left(\theta_{0}, \rho_{0}\right)$ lies in this open ball, then $\left(\theta_{k}, \rho_{k}\right)$ lies in the ball for all $k$ and $\rho_{k} \rightarrow 0$ exponentially fast.

Proof. The time-discretized version of Malkin structure takes the following form

$$
\left[\begin{array}{l}
\theta_{k+1} \\
\rho_{k+1}
\end{array}\right]=\left(1+\epsilon\left[\begin{array}{ll}
0 & 0 \\
0 & A
\end{array}\right]\right)\left[\begin{array}{c}
\theta_{k} \\
\rho_{k}
\end{array}\right]+\epsilon\left[\begin{array}{c}
\Theta\left(\theta_{k}, \rho_{k}\right) \\
P\left(\theta_{k}, \rho_{k}\right)
\end{array}\right]
$$

where $A$ has eigenvalues with negative real parts, $\Theta(\theta, 0)=0$ and $P(\theta, 0)=0$. Define

$$
\begin{array}{r}
h_{1}\left(\theta_{k}\right)=\lim _{\rho_{k} \rightarrow 0} \frac{\Theta\left(\theta_{k}, \rho_{k}\right)}{\left\|\rho_{k}\right\|}, \quad h_{2}\left(\theta_{k}\right)=\lim _{\rho_{k} \rightarrow 0} \frac{P\left(\theta_{k}, \rho_{k}\right)}{\left\|\rho_{k}\right\|}, \\
b_{1}=\left\{\begin{array}{ll}
\frac{\Theta\left(\theta_{k}, \rho_{k}\right)}{\left\|\rho_{k}\right\|} & \text { if } \rho_{k} \neq 0 \\
h_{1}\left(\theta_{k}\right) & \text { if } \rho_{k}=0
\end{array} \quad, b_{2}= \begin{cases}\frac{P\left(\theta_{k}, \rho_{k}\right)}{\left\|\rho_{k}\right\|} & \text { if } \rho_{k} \neq 0 \\
h_{2}\left(\theta_{k}\right) & \text { if } \rho_{k}=0\end{cases} \right.
\end{array}
$$

Because $\lim _{\rho \rightarrow 0} \frac{P(0, \rho)}{\|\rho\|}=0$, we know that $b_{2}(0)=0$. Since $A$ has eigenvalues with negative real parts, for all sufficiently small $\tau>0$, the matrix $A_{d}:=I+\tau A$ will have eigenvalues inside the unit circle. Without loss of generality, we may assume (using a nonsingular similarity transformation $T$ if necessary, corresponding to a replacement of $\rho_{k}$ by $T \rho_{k}$ ) that for some $\gamma>0$, there holds

$$
I-A_{d}^{\top} A_{d} \geq \gamma I
$$

Now set $V\left(\rho_{k}\right)=\rho_{k}^{\top} \rho_{k}$. Also, note that given any $\sigma>0$, there exists $\eta(\sigma)$ and a closed ball $\overline{\mathcal{B}}_{\eta}$, without loss of generality contained in $\mathcal{V}$, such that

$$
\left\|b_{1}\left(\rho_{k}, \theta_{k}\right)\right\| \leq \sigma \forall\left(\rho_{k}, \theta_{k}\right) \in \overline{\mathcal{B}}_{\eta}
$$

Now observe that for $\left(\rho_{k}, \theta_{k}\right) \in \mathcal{B}_{\eta}$ there holds

$$
\begin{aligned}
& V\left(\rho_{k+1}\right)-V\left(\rho_{k}\right) \\
= & \rho_{k}^{\top}\left(I-A_{d}^{\top} A_{d}\right) \rho_{k}+2 \tau \rho_{k}^{\top} A_{d}^{\top} P\left(\rho_{k}, \theta_{k}\right)+\tau^{2}\left\|P\left(\rho_{k}, \theta_{k}\right)\right\|^{2} \\
\leq \quad & -\gamma \rho_{k}^{\top} \rho_{k}+2 \tau\left\|A_{d}\right\|\left\|\rho_{k}\right\|^{2}\left\|b_{1}\left(\rho_{k}, \theta_{k}\right)\right\| \\
& +\tau^{2}\|\| \rho_{k}\left\|^{2}\right\| b_{1}\left(\rho_{k}, \theta_{k}\right) \|^{2} \\
\leq \quad & \left(-\gamma+2 \tau \sigma+\tau^{2} \sigma^{2}\right)\left\|\rho_{k}\right\|^{2} \\
\leq \quad & \left(-\gamma+2 \sigma+\sigma^{2}\right)\left\|\rho_{k}\right\|^{2}
\end{aligned}
$$

Restrict $\sigma$ to be small enough that $2 \sigma+\sigma^{2}<\gamma / 2$. Then we achieve:

$$
V\left(\rho_{k+1}\right)-V\left(\rho_{k}\right) \leq-(\gamma / 2) V\left(\rho_{k}\right)
$$

and

$$
\left\|\rho_{k+1}\right\|^{2} \leq(1-(\gamma / 2))\left\|\rho_{k}\right\|^{2}
$$

Provided that the sequence $\left(\rho_{k}, \theta_{k}\right)$ remains in $\mathcal{B}_{\eta}$, exponential convergence to zero of $\rho_{k}$ is achieved. We shall now argue that this can be assured through appropriate selection of the initial condition. Suppose to obtain a contradiction that there exists a finite $K$ such that $\left(\rho_{k}, \theta_{k}\right) \in \mathcal{B}_{\eta} \forall k \in[0, K]$ but the condition fails for $k=K+1$. Suppose that the function $b_{2}$, which is continuous, attains an upper bound of $\bar{m}$ on $\overline{\mathcal{B}}_{\eta}$. Observe that for all $k \in[0, K]$,

$$
\begin{aligned}
\left\|\Theta\left(\rho_{k}, \theta_{k}\right)\right\| & =\left\|\rho_{k}\right\|\left\|b_{2}\left(\rho_{k}, \theta_{k}\right)\right\| \\
& \leq \bar{m}\left\|\rho_{0}\right\|(1-(\gamma / 2))^{k}
\end{aligned}
$$

which implies by summation that

$$
\left\|\theta_{k+1}\right\| \leq \bar{m}\left\|\rho_{0}\right\| \frac{1}{1-(\gamma / 2)}+\left\|\theta_{0}\right\|
$$

Now restrict the initial condition $\left(\rho_{0}, \theta_{0}\right)$ to lie-in a smaller ball than $\mathcal{B}_{\eta}$. Define a $\eta_{0}<\eta$ as a positive quantity satisfying

$$
\eta_{0}+\bar{m} \frac{1}{1-(\gamma / 2)} \eta_{0}+\eta_{0}<\eta
$$

and suppose that $\left(\rho_{0}, \theta_{0}\right) \in \mathcal{B}_{\eta_{0}}$. Then while the trajectory $\left(\rho_{k}, \theta_{k}\right)$ remains in $\mathcal{B}_{\eta}$, i.e. for all $k \in[0, K]$ with $K$ maximal, we know using (35), (37) that

$$
\begin{aligned}
& \left\|\left(\rho_{k+1}, \theta_{k+1}\right)\right\| \leq\left\|\rho_{k+1}\right\|+\left\|\theta_{k+1}\right\| \\
\leq \quad & \eta_{0}+\bar{m} \frac{1}{1-(\gamma / 2)} \eta_{0}+\eta_{0}<\eta
\end{aligned}
$$

This shows that $\left(\rho_{K+1}, \theta_{K+1}\right) \in \mathcal{B}_{\eta}$, and that $K$ is not maximal, i.e. there cannot be a finite $K$. Hence exponential convergence of the sequence $\rho_{k}$ and convergence of the sequence $\theta_{k}$ is established.

Theorem 3. Consider the system of equations in (15) and suppose the graph associated with the velocity measurements is connected and undirected. Then (15) is asymptotically stable for sufficiently small $\epsilon_{1}$ and $\epsilon_{2}$.

Proof. To show the stability of (15) for suitable $\epsilon_{i}$, we initially study certain variants on (17) and examine their stability. First, if the second equation of (17) is replaced for some positive $\alpha, \beta$ by

$$
\dot{\bar{v}}=\alpha L \bar{v}+\beta \bar{f}(\bar{p})
$$

the convergence properties are unaffected. Of course, the speed of convergence is changed.

Second, if (40) is replaced for any $\epsilon>0$ by

$$
\begin{aligned}
& \dot{\hat{p}}=\hat{v} \\
& \dot{\hat{v}}=\epsilon \alpha L \hat{v}+\epsilon^{2} \beta \bar{f}(\hat{p})
\end{aligned}
$$


or alternatively by

$$
\begin{aligned}
\dot{\hat{p}} & =\epsilon \hat{w} \\
\dot{\hat{w}} & =\epsilon \alpha L \hat{w}+\epsilon \beta \bar{f}(\hat{p})
\end{aligned}
$$

then any solution of (40) gives rise to solutions of (41) and (42) and vice versa through

$$
\left[\begin{array}{c}
\bar{p}(\epsilon t) \\
\epsilon \bar{v}(\epsilon t)
\end{array}\right]=\left[\begin{array}{c}
\hat{p}(t) \\
\hat{v}(t)
\end{array}\right]=\left[\begin{array}{c}
\hat{p}(t) \\
\epsilon \hat{w}(t)
\end{array}\right]
$$

The discrete-time equation with which we are working in (15) is a discretization of (41) (after some transformation and with appropriate identification of $\epsilon_{1}, \epsilon_{2}$ ). Now if the original equation (40) is approximated by a difference equation with sampling interval $h$, this is equivalent to sampling (42) with sampling interval $h / \epsilon$, or sampling (41) with the same sampling interval. In particular, if $h$ is such that discretization of (40) gives solutions which converge exponentially fast to the center manifold associated with that equation, then with discretization interval $h / \epsilon$, solutions of the discretized version of (41) or (42) will also converge exponentially fast to the center manifold. In particular, if $\epsilon$ is chosen so that $h / \epsilon=T$, then for that value of $\epsilon$ and with the sampling interval $T$, the desired convergence will occur. In summary, if $\alpha, \beta$ are prescribed, and if a sampling interval $h$ is chosen so that the discretized version of (40) converges to the center manifold, then taking $\epsilon=h / T, \epsilon_{1}=\alpha \epsilon, \epsilon_{2}=\beta \epsilon^{2}$ will be satisfactory in (15). Of course, $\alpha=\beta=1$ is legitimate; with $\epsilon$ small, the values of $\epsilon_{1}, \epsilon_{2}$ will be such that velocity consensus is effectively achieved before the correct shape. This is intuitively reasonable.

So the question arises as to whether discretization of (40) with a sufficiently small sampling interval will give convergence. Lemma 2 shows that (17) can be transformed to a Malkin structure and therefore (40). Furthermore, Theorem 2 shows that the discretization of Malkin structure with a sufficiently small sampling interval will give convergence. In fact the operations of coordinate basis change through a diffeomorphism to a Malkin equation and timediscretization commute (see Appendix III of [16]). Therefore, the theorem is proved.

\section{B. Simulation Results combining Velocity Consensus and formation shape control}

Consider a three-agent system where each agent can measure its distance to the other two agents. The goal is to achieve velocity consensus and form a triangular formation. Suppose $\omega_{i}$ is the angular velocity of agent $i, T$ is the sampling time interval, $\left(v_{x i}, v_{y i}\right)$ is the translational velocity of agent $i$ and $\left(p_{x i}, p_{y i}\right)$ is the position of circle center of agent $i$. In the simulation, we set $\omega_{1}=5$, $\omega_{2}=-3, \omega_{3}=7, T=2 \pi$. When $t=0,\left(v_{x 1}, v_{y 1}\right)=(-4,1.5)$, $\left(v_{x 2}, v_{y 2}\right)=(3,-3.5),\left(v_{x 3}, v_{y 3}\right)=(2,3.5),\left(p_{x 1}, p_{y 1}\right)=$ $(100,50),\left(p_{x 2}, p_{y 2}\right)=(0,80),\left(p_{x 3}, p_{y 3}\right)=(0,0), \epsilon_{1}=5 \times 10^{-2}$ and $\epsilon_{2}=7 \times 10^{-7}$. The desired distance between each pair of agents in the formation is 20 . The trajectories of the agents are shown in Fig. 5.

\section{CONCLUSION}

In this paper, we proposed a strategy to achieve velocity consensus and formation control using distance-only measurements for multiple agents. Given the fact that for agents to execute arbitrary motions, instantaneous distance-only measurements cannot provide enough information for achieving velocity consensus and formation control, we studied agents performing a combination of circular motion and linear motion.

In further research, we are looking to achieve formation control and velocity consensus using agent perturbations, where agents are

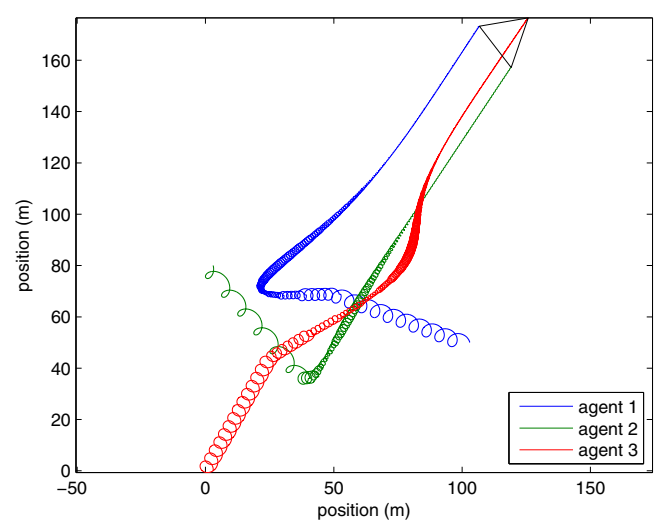

Fig. 5. Result of combining velocity consensus and formation shape control using distance-only measurements with adaptive radius setting: The trajectories of agents. Triangular formation and velocity consensus are achieved.

not limited to performing a combination of circular motion and linear motion. In addition, it appears very likely that the same strategy as we proposed in this paper can be used in velocity consensus using bearing-only measurements.

\section{REFERENCES}

[1] W. Ren, R. W. Beard, and E. M. Atkins, "Information consensus in multivehicle cooperative control," IEEE Control Systems, vol. 27, no. 2, pp. 71-82, 2007.

[2] B. D. O. Anderson, C. Yu, B. Fidan, and J. Hendrickx, "Rigid graph control architectures for autonomous formations," IEEE Control Systems, vol. 28, no. 6, pp. 48-63, 2008.

[3] H. Wymeersch, J. Lien, and M. Z. Win, "Cooperative localization in wireless networks," Proceedings of the IEEE, vol. 97, no. 2, pp. 427450, 2009.

[4] G. Foderaro and S. Ferrari, "Necessary conditions for optimality for a distributed optimal control problem," in 49th IEEE Conference on Decision and Control (CDC). IEEE, 2010, pp. 4831-4838.

[5] B. Jiang, Z. Sun, and B. Anderson, "Higher order voronoi based mobile coverage control," in American Control Conference (ACC), 2015, pp. 1457-1462.

[6] B. D. O. Anderson and C. Yu, "Range-only sensing for formation shape control and easy sensor network localization," in Chinese Control and Decision Conference (CCDC). IEEE, 2011, pp. 3310-3315.

[7] T. H. Summers, C. Yu, S. Dasgupta, and B. D. O. Anderson, "Control of minimally persistent leader-remote-follower and coleader formations in the plane," IEEE Transactions on Automatic Control, vol. 56, no. 12, pp. 2778-2792, 2011.

[8] M. Cao, C. Yu, and B. D. O. Anderson, "Formation control using rangeonly measurements," Automatica, vol. 47, no. 4, pp. 776-781, 2011.

[9] Y. Zhu, S. Gortler, and D. Thurston, "Sensor network localization using sensor perturbation," ACM Transactions on Sensor Networks (TOSN), vol. 7, no. 4, p. 36, 2011.

[10] Y.-P. Tian and Q. Wang, "Global stabilization of rigid formations in the plane," Automatica, vol. 49, no. 5, pp. 1436-1441, 2013.

[11] B. Jiang, M. Deghat, and B. Anderson, "Translational velocity consensus using distance-only measurements," in 52nd IEEE Conference on Decision and Control (CDC), Dec 2013, pp. 2746-2751.

[12] B. D. Anderson, Z. Lin, and M. Deghat, "Combining distance-based formation shape control with formation translation," Developments in Control Theory Towards Glocal Control, Eds. L. Qiu, J. Chen, T. Iwasaki and H. Fujioka, pp. 121-130, 2012.

[13] L. Krick, "Application of graph rigidity in formation control of multirobot networks," Master's thesis, University of Toronto, 2007.

[14] L. Krick, M. E. Broucke, and B. A. Francis, "Stabilization of infinitesimally rigid formations of multi-robot networks," International Journal of Control, vol. 82, no. 3, pp. 423-439, 2009.

[15] M. Deghat, B. D. O. Anderson, and Z. Lin, "Combined flocking and distance-based shape control of multi-agent formations," Submitted for publication in IEEE Transactions on Automatic Control, 2015.

[16] B. Jiang, M. Deghat, and B. D. O. Anderson, "Velocity consensus and formation shape control using distance-only measurements," CoRR, vol. abs/1411.3841, 2014. [Online]. Available: http://arxiv.org/abs/1411.3841 